\title{
Influência da prematuridade e do baixo peso ao nascimento sobre a função pulmonar na idade escolar: uma revisão de literatura
}

\author{
Influence of prematurity and low birth weight on lung function at school age: a literature review
}

\author{
Aline Dill Winck ${ }^{a}$, João Paulo Heinzmann-Filho ${ }^{b}$, Suelen Goecks Oliveirac, Renato Tetelbom Stein ${ }^{\mathrm{d}}$ \\ a Fisioterapeuta. Doutora em Pediatria e Saúde da Criança pela Pontifícia Universidade Católica do Rio Grande do Sul (PUCRS). \\ Professora da Universidade de Caxias do Sul (UCS) e do Centro Universitário Metodista (IPA). \\ b Fisioterapeuta. Doutor em Pediatria e Saúde da Criança (PUCRS). Pesquisador do Programa de Pós-Graduação em Saúde da Criança (PUCRS). \\ Enfermeira. Mestre em Pediatria e Saúde da Criança (PUCRS) \\ ¿ Eneumologista Pediátrico. Doutor em Ciências Pneumológicas pela Universidade Federal do Rio Grande do Sul (UFRGS). Professor e Coordenador \\ do Programa de Pós-Graduação em Pediatria e Saúde da Criança (PUCRS).
}

RESUMO

Objetivo: Avaliar, através de uma revisão de literatura, a influência da prematuridade e do baixo peso ao nascimento sobre a função pulmonar na idade escolar.

Materiais e Métodos: Trata-se de uma revisão integrativa. Foram utilizadas as bases de dados Lilacs, Pubmed, Scopus e Scielo, selecionando estudos observacionais ou ensaios clínicos, publicados em inglês ou português, que objetivassem avaliar a função pulmonar (espirometria) na idade escolar de prematuros com baixo peso ao nascimento em comparação com crianças nascidas a termos com peso adequado. As palavras-chave e os operadores boleanos utilizados foram: Infant, Extremely Premature AND Respiratory Function Tests OR Forced Expiratory Volume in 1 second OR FVC OR Forced Vital Capacity OR 2575 percent, FeF OR Forced mid-expiratory flow OR FEV1 OR Forced Expiratory volume AND Very low birth weight.

Resultados: De um total de 1032 artigos encontrados, 15 foram selecionados para inclusão final no presente estudo. Grande parte dos estudos (33,3\%) foi realizada na Austrália, sendo que nenhum foi localizado na população brasileira. O tamanho amostral dos artigos variou de 27 até 2150 indivíduos entre cada grupo estudado. A idade gestacional e o baixo peso ao nascimento do grupo de prematuros foram classificados como abaixo de 37 semanas e 53,3\% avaliaram recém-nascidos de muito baixo peso. A idade dos escolares, no momento da avaliação da função pulmonar, variou entre 5 e 14 anos, sendo que a maioria, 12 (80,0\%), apresentou redução da função pulmonar no grupo de crianças prematuras e com baixo peso, enquanto 3 (20\%) dos trabalhos apontaram valores espirométricos normais.

Conclusão: Os resultados demonstram que a prematuridade e o baixo peso ao nascimento parecem influenciar de maneira negativa sobre a função pulmonar das crianças na idade escolar.

Palavras-chave: prematuro; peso ao nascer; testes de função respiratória; pediatria. observational studies or clinical trials published in English or Portuguese, which aimed to evaluate pulmonary function (spirometry) at school age of preterm with low birth weight compared to children born on term with normal weight. The keywords and boolean operators used were: Infant, Extremely Premature AND Respiratory Function Tests Forced Expiratory Volume in OR 1 OR second FVC Forced Vital Capacity OR OR 2575 percent, FeF OR Forced expiratory flow mid-OR FEV1 Forced Expiratory volume AND OR Very low birth weight.

Results: From a total of 1032 articles found, 15 were selected for final inclusion in this study. Most studies (33.3\%) were performed in Australia, and none was found in the Brazilian population. The sample size of the studies ranged from 27 to 2150 individuals for each group studied. The gestational age and the low birth weight in the premature group were classified as less than 37 weeks and $53.3 \%$ rated newborns as very low weight. The age of the scholars during the evaluation of lung function ranged from 5 to 14 years, with the majority (80.0\%) presenting reduced lung function in the premature with low weight children, while $3(20 \%)$ of the publications indicated normal spirometric values.

Conclusion: The results show that prematurity and low birth weight appears to adversely impact lung function of children at school age.

Keywords: premature; birth weight; respiratory function tests; pediatrics. 


\section{INTRODUÇÃO}

A idade gestacional e o peso ao nascimento são importantes indicadores de risco biológico para problemas de desenvolvimento da criança $^{1,2}$. As crianças nascidas prematuras estão mais propensas ao desenvolvimento de agravos futuros, como consequência da pouca maturidade dos órgãos e de danos oriundos da associação com o baixo peso ao nascimento ${ }^{3}$.

Os avanços da terapia médica, associados com a introdução de intervenções, como surfactante e esteroides pré-natais, fizeram com que a taxa de sobrevivência de recém-nascidos de muito baixo peso aumentasse na última década ${ }^{4}$. Apesar desse aumento, tem-se observado que longos períodos de suplementação de oxigênio ou ventilação mecânica podem causar danos pulmonares irreversíveis e contribuir para o desenvolvimento de morbidades respiratórias ao longo da infância ${ }^{5}$. Um estudo prévio, que avaliou RNMBP, demonstrou uma frequência maior de doenças e readmissões hospitalares nos primeiros 2 anos de vida, quando comparados a um grupo de crianças com peso normal ao nascimento ${ }^{6}$. Embora esses comprometimentos tornam-se evidentes na literatura, outros achados demonstram que as alterações observadas no primeiro ano de vida podem ser recuperadas ou vir a tornar-se menos importantes no decorrer da vida ${ }^{7,8}$.

Diversos estudos vêm sendo publicados com o intuito de avaliar os efeitos da prematuridade e do baixo peso ao nascimento sobre a função pulmonar ao longo da infância, principalmente na idade escolar ${ }^{9-12}$. Além disso, muitos desses estudos buscam entender a influência das variáveis do período perinatal/neonatal sobre a condição respiratória desses sujeitos ${ }^{13-15}$. No entanto, ainda encontram-se incertezas sobre a função pulmonar dessas crianças na idade escolar, variando desde achados de redução da função respiratória até valores de normalidade ${ }^{8}$.

Portanto, considerando os achados contraditórios sobre a influência da prematuridade e do baixo peso ao nascimento sobre as alterações na função pulmonar no decorrer da infância, o objetivo desta revisão foi avaliar a influência da prematuridade e do baixo peso sobre a função pulmonar na idade escolar. O melhor conhecimento sobre o tema pode colaborar para um melhor entendimento da condição respiratória nessa população, visando o desenvolvimento de medidas efetivas para amenizar as morbidades e os comprometimentos respiratórios ao decorrer da vida.

\section{MATERIAIS E MÉTODOS}

Esse estudo consiste em uma revisão integrativa realizada por meio de pesquisa nas bases de dados Pubmed, Scopus,
Lilacs e Scielo. Foram selecionados estudos nos idiomas português e inglês, que estivessem disponíveis na íntegra e que as palavras-chaves estivessem presentes no título e/ou resumo. Não foram utilizados filtros em relação ao ano de publicação dos estudos. As palavras-chaves e os operadores boleanos utilizados nas bases de dados foram: Infant, Extremely Premature AND Respiratory Function Tests OR Forced Expiratory Volume in 1 second OR FVC OR Forced Vital Capacity OR 2575 percent, FeF OR Forced mid-expiratory flow OR FEV1 OR Forced Expiratory volume AND Very low birth weight. As buscas foram realizadas entre o período de julho a outubro de 2014.

Foram utilizados, como critérios de inclusão, estudos observacionais, do tipo transversal, caso-controle, coorte e retrospectivo ou ensaios clínicos, que objetivassem avaliar a função pulmonar por meio da espirometria, em escolares prematuros e com baixo peso ao nascimento, em comparação com crianças nascidas a termo e com peso adequado. As definições da idade gestacional e do peso ao nascimento foram estabelecidas de acordo com os critérios de cada autor. Além disso, a avaliação da função pulmonar (espirometria) foi considerada normal ou reduzida de acordo com a classificação dos próprios autores. Em contrapartida, foram excluídos estudos de revisão, meta-análise e aqueles que não realizaram a comparação da função pulmonar na idade escolar com um grupo de crianças nascidas a termo e com peso adequado.

Primeiramente, foi realizada a avaliação dos títulos e dos resumos (abstracts) para possível inclusão. Os estudos que contemplaram os critérios de inclusão foram selecionados para leitura na íntegra e posteriormente sumarizados em uma tabela para melhor visualização dos resultados. Foram registradas as seguintes características dos estudos: nome do primeiro autor e ano de publicação do estudo, país da coleta de dados, tamanho amostral, grupos analisados, idade gestacional, peso ao nascimento, idade atual das crianças no dia da avaliação pulmonar e os resultados da função pulmonar.

\section{RESULTADOS}

De um total de 1032 artigos encontrados nas bases de dados, 31 foram selecionados para leitura na íntegra. Desses, 15 foram selecionados para a inclusão final no presente estudo. A Figura 1 apresenta a estratégia de seleção dos estudos identificados na presente busca.

Grande parte dos estudos foi realizada na Austrália $(33,3 \%)$ e na Finlândia (13,3\%), sendo que nenhum estudo foi localizado na população brasileira. Esses estudos foram publicados entre os anos de 1990 e 2013. Os artigos incluídos apresentaram uma grande variabilidade sobre o tamanho da 
amostra, variando de 27 até 2150 indivíduos. Em relação à classificação da idade gestacional do grupo de prematuros e de baixo peso, foram incluídas crianças nascidas com $<37$ até $<28$ semanas e a maioria dos estudos $(53,3 \%)$ avaliou recém-nascidos de muito baixo peso ( $\leq 1500$ gramas). Quando se analisou esses aspectos no grupo de recémnascidos controles, a classificação da idade gestacional desse grupo foi maior que 36 semanas e o peso ao nascimento, maior do que 2220 gramas.

A idade dos escolares, no momento da avaliação da função pulmonar, variou de 5 a 14 anos, sendo que 3 estudos apresentaram valores normais de função pulmonar nas crianças prematuras e com baixo peso, enquanto 12 estudos apresentaram redução de, pelo menos, alguma variável espirométrica. Destas, as mais frequentes foram o $\operatorname{VEF}_{1}(66,6 \%)$ e a CVF (60\%). A sumarização das características dos estudos incluídos é demonstrada na Tabela 1.

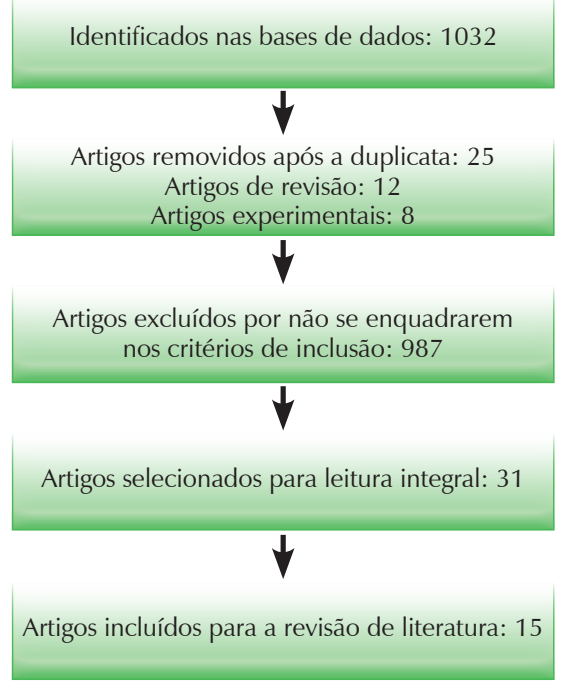

Figura 1. Estratégia de seleção para a inclusão dos artigos.

Tabela 1 - Características dos estudos incluídos no presente estudo.

\begin{tabular}{|c|c|c|c|c|c|c|c|}
\hline $\begin{array}{l}\text { Autor } \\
\text { Ano }\end{array}$ & País & $\begin{array}{l}\text { Tamanho } \\
\text { amostral }\end{array}$ & Grupos analisados & $\begin{array}{l}\text { Idade gestacional } \\
\text { (semanas) }\end{array}$ & $\begin{array}{l}\text { Peso ao } \\
\text { nascimento } \\
\text { (gramas) }\end{array}$ & $\begin{array}{l}\text { Idade da } \\
\text { avaliação }\end{array}$ & $\begin{array}{l}\text { Resultados da } \\
\text { função pulmonar }\end{array}$ \\
\hline $\begin{array}{l}\text { Chan et al. } \\
1990^{16}\end{array}$ & Inglaterra & $\begin{array}{l}27 \\
46\end{array}$ & $\begin{array}{l}\text { RNMBP } \\
\text { RNC }\end{array}$ & $\begin{array}{l}\leq 35 \\
>36\end{array}$ & $\begin{array}{l}\leq 1500 \\
>2200\end{array}$ & 9 anos & $\begin{array}{c}\text { Redução do } \\
\text { VEF }_{1} \text { e CVF nos RNMBP }\end{array}$ \\
\hline $\begin{array}{l}\text { Kitchenet al. } \\
1992^{6}\end{array}$ & Austrália & $\begin{array}{l}74 \\
96 \\
53\end{array}$ & $\begin{array}{l}\text { RNEBP } \\
\text { RNMBP } \\
\text { RNC }\end{array}$ & $\begin{array}{l}27,4 \pm 2,1 \\
29,6 \pm 1,5 \\
\quad \geq 37\end{array}$ & $\begin{array}{c}500-999 \\
1000-1500 \\
>2500\end{array}$ & 8 anos & Similar nos três grupos \\
\hline $\begin{array}{l}\text { McLeodet al. } \\
1996^{14}\end{array}$ & Escócia & $\begin{array}{l}292 \\
574\end{array}$ & $\begin{array}{l}\text { RNMBP } \\
\text { RNC }\end{array}$ & $\begin{array}{l}<37 \\
\geq 37\end{array}$ & $\begin{array}{l}<1500 \\
>2500\end{array}$ & 8-9 anos & $\begin{array}{c}\text { Redução de } \\
\text { CVF nos RNMBP }\end{array}$ \\
\hline $\begin{array}{l}\text { Lercheret al. } \\
1997^{17}\end{array}$ & Áustria & $\begin{array}{c}45 \\
599\end{array}$ & $\begin{array}{l}\text { RNBP } \\
\text { RNC }\end{array}$ & $\begin{array}{l}<37 \\
\geq 37\end{array}$ & $\begin{array}{l}<2500 \\
\geq 2500\end{array}$ & 7-12 anos & Similar nos dois grupos \\
\hline $\begin{array}{l}\text { Schraederet al. } \\
1998^{18}\end{array}$ & Filadélfia & $\begin{array}{l}30 \\
30\end{array}$ & $\begin{array}{l}\text { RNMBP } \\
\text { RNC }\end{array}$ & $\begin{array}{l}<37 \\
\geq 37\end{array}$ & $\begin{array}{l}\leq 1500 \\
>2500\end{array}$ & 10-11 anos & $\begin{array}{c}\text { Redução de } \\
\text { FEF }_{25-75 \%} \text { nos RNMBP }\end{array}$ \\
\hline $\begin{array}{l}\text { Wjstet al. } \\
1998^{19}\end{array}$ & Alemanha & $\begin{array}{c}187 \\
2150\end{array}$ & $\begin{array}{l}\text { RNPT baixo/ } \\
\text { adequado peso } \\
\text { RNT baixo/ } \\
\text { adequado peso }\end{array}$ & $\begin{array}{l}<38 \\
\geq 30\end{array}$ & $\begin{array}{l}<2500 / \geq 2500 \\
<2500 / \geq 2500\end{array}$ & 5-14 anos & $\begin{array}{c}\text { Redução do } \\
\text { VEF }_{1} \text { e CVF nos RNPT } \\
\text { com baixo peso }\end{array}$ \\
\hline $\begin{array}{l}\text { Kennedy et al. } \\
2000^{20}\end{array}$ & Austrália & $\begin{array}{c}102 \\
82\end{array}$ & $\begin{array}{l}\text { RNMBP com e sem } \\
\text { BDP } \\
\text { RNC }\end{array}$ & $\begin{array}{l}29,6 \pm 2,8 \\
40,0 \pm 1,6\end{array}$ & $\begin{array}{l}\leq 1500 \\
>2500\end{array}$ & 11 anos & $\begin{array}{c}\text { Redução de } \\
\text { VEF }_{1}, \text { CVF e FEF } \\
\text { nos RNMBP }\end{array}$ \\
\hline $\begin{array}{l}\text { Siltanenet al. } \\
2004^{9}\end{array}$ & Finlândia & $\begin{array}{l}72 \\
65\end{array}$ & $\begin{array}{l}\text { RNMBP } \\
\text { RNC }\end{array}$ & $\begin{array}{l}<34 \\
\geq 37\end{array}$ & $\begin{array}{l}<1501 \\
>2500\end{array}$ & 10 anos & $\begin{array}{c}\text { Redução de } \\
\mathrm{VEF}_{1}, \mathrm{CVF}, \mathrm{CVF} / \mathrm{VEF}_{1}, \\
\mathrm{FEF}_{50 \%} \text { e } \mathrm{FEF}_{25-75 \%} \\
\text { nos RNMBP }\end{array}$ \\
\hline $\begin{array}{l}\text { Korhonenet al. } \\
2004^{21}\end{array}$ & Finlândia & $\begin{array}{l}68 \\
34\end{array}$ & $\begin{array}{l}\text { RNMBP com e sem } \\
\text { DBP } \\
\text { RNC }\end{array}$ & $\begin{array}{l}<37 \\
\geq 37\end{array}$ & $\begin{array}{l}<1500 \\
>2500\end{array}$ & 7-8 anos & $\begin{array}{c}\text { Redução de } \\
\mathrm{VEF}_{1} \text { nos RNMBP } \\
\text { com DBP }\end{array}$ \\
\hline Doyle et al. $2006^{22}$ & Austrália & $\begin{array}{l}298 \\
262\end{array}$ & $\begin{array}{c}\text { RNEBP } \\
\text { RNC }\end{array}$ & $\begin{array}{l}<28 \\
\geq 37\end{array}$ & $\begin{array}{l}<1000 \\
>2499\end{array}$ & 8-9 anos & $\begin{array}{c}\text { Redução de } \\
\text { VEF }_{1}, \mathrm{CVFF}_{\text {, VEF }} / \mathrm{CVFF}_{\text {e }} \mathrm{FEF}_{25-75 \%} \\
\text { nos RNEBP }\end{array}$ \\
\hline $\begin{array}{l}\text { Paltaet al. } \\
2007^{23}\end{array}$ & Estados Unidos & $\begin{array}{l}265 \\
360\end{array}$ & $\begin{array}{l}\text { RNMBP com e sem } \\
\text { DBP } \\
\text { RNC }\end{array}$ & $\begin{array}{l}29,0 \pm 2,5 \\
29,0 \pm 2,6\end{array}$ & $\begin{array}{l}<1500 \\
>2500\end{array}$ & 10 anos & $\begin{array}{c}\text { Redução do } \\
V_{E F_{1}} \text { e CVF e PFE }\end{array}$ \\
\hline $\begin{array}{l}\text { Smith et al. } \\
2008^{24}\end{array}$ & Austrália & $\begin{array}{c}127 \\
26\end{array}$ & $\begin{array}{l}\text { RNEBP } \\
\text { RNC }\end{array}$ & $\begin{array}{l}<32 \\
>37\end{array}$ & $\begin{array}{l}<1000 \\
>2500\end{array}$ & 10 anos & $\begin{array}{c}\text { Redução de } \\
\text { VEF }, \text { CVF e } \\
\mathrm{FEF}_{25-75 \%} \text { nos RNEBP }\end{array}$ \\
\hline $\begin{array}{l}\text { He-Q et al. } \\
2009^{25}\end{array}$ & China & $\begin{array}{c}117 \\
1472\end{array}$ & $\begin{array}{l}\text { RNBP } \\
\text { RNC }\end{array}$ & $\begin{array}{l}<37 \\
\geq 37\end{array}$ & $\begin{array}{l}\leq 2500 \\
>2500\end{array}$ & 9-11 anos & Similar nos dois grupos \\
\hline $\begin{array}{l}\text { Cazzato et al. } \\
2013^{26}\end{array}$ & Itália & $\begin{array}{l}48 \\
46\end{array}$ & $\begin{array}{l}\text { RNMBP com e sem } \\
\text { DBP } \\
\text { RNC }\end{array}$ & $\begin{array}{l}\leq 32 \\
\geq 37\end{array}$ & $\begin{array}{l}\leq 1500 \\
>2500\end{array}$ & 8-9 anos & $\begin{array}{c}\text { Redução do } \\
\text { CVF, VEF } \text { e }_{1} \\
\text { FEF }_{25-75 \%} \text { RNMBP }^{\text {RNMB }}\end{array}$ \\
\hline $\begin{array}{l}\text { Hacking et al. } \\
2013^{27}\end{array}$ & Austrália & $\begin{array}{l}240 \\
208\end{array}$ & $\begin{array}{l}\text { RNEBP com DBP } \\
\text { RNC }\end{array}$ & $\begin{array}{l}<28 \\
>36\end{array}$ & $\begin{array}{l}<1000 \\
>2499\end{array}$ & 8-9 anos & $\begin{array}{l}\text { Redução do } \\
\text { VEF }_{1} \text { e } \mathrm{FEF}_{25-75 \%} \\
\text { nos RNEBP }\end{array}$ \\
\hline
\end{tabular}

Grupos analisados: recém-nascidos de extremo baixo peso (RNEBP); recém-nascidos de muito baixo peso (RNMBP); recém-nascidos do grupo controle (RNC); recém-nascidos a termo (RNT); recém-nascidos prematuros (RNPT); displasia bronco pulmonar (DBP). Resultado da função pulmonar: capacidade vital forçada (CVF); volume expiratório forçado no primeiro segundo (VEF1); fluxo expiratório forçado entre 25 e $75 \%$ da CVF ( $\mathrm{FEF}_{25-75 \%}$ ); fluxo expiratório forçado em 50\% da CVF (FEF50\%); pico de fluxo expiratório (PFE). 


\section{DISCUSSÃO}

Na presente revisão, foram identificados 15 estudos que avaliaram a influência da prematuridade e do baixo peso ao nascimento sobre a função pulmonar de crianças na idade escolar. Dos 15 artigos incluídos, a grande maioria ${ }^{9,14,16,18-}$ $24,26,27$ dos estudos identificou redução da função pulmonar na idade escolar no grupo estudado, enquanto somente $3^{6,17,25}$ encontraram valores dentro dos padrões de normalidade em crianças nascidas prematuras e com baixo peso, quando comparadas com a termo/peso adequado.

Para fins de verificação da função pulmonar, todos os estudos utilizaram o método da espirometria, associados com outro tipo de teste de função pulmonar, não avaliado nessa revisão. Esses métodos combinados variaram desde a mensuração da inflamação das vias aéreas, por meio do óxido nítrico exalado, até a quantificação de volumes pulmonares, através da pletismografia corporal. O método de escolha para avaliação funcional nessa população deve apresentar boa sensibilidade para a detecção da redução de fluxos expiratórios, tendo em vista que os distúrbios ventilatórios predominantes nas enfermidades crônicas decorrentes da prematuridade são, em sua maioria, obstrutivos ${ }^{6,28}$. Desta forma, pode-se considerar que a espirometria é o teste mais utilizado com esse propósito em crianças e adultos.

Os resultados dessa revisão demonstraram que a prematuridade e o baixo peso ao nascimento causam efeitos negativos sobre a função pulmonar em crianças na idade escolar. Tais achados podem ser atribuídos ao fato de que a idade gestacional e o peso de nascimento parecem ser mais determinantes da função pulmonar na infância do que as intercorrências durante o período perinatal. Além disso, o retardo de crescimento intrauterino causado por essas duas condições pode levar a um padrão discordante do crescimento pulmonar e das vias aéreas, fazendo com que as vias aéreas não acompanhem o desenvolvimento normal do parênquima pulmonar no período pós-natal, resultando na redução persistente de fluxos expiratórios ${ }^{16,29}$. Dos estudos que apresentaram redução da função pulmonar, 5 incluíram um grupo de recém-nascidos com displasia broncopulmonar (DBP), comparando-os com crianças que não apresentavam a doença ou ainda com grupos de nascidos a termo/peso adequado $20,21,23,26,27$, o que pode ter colaborado para o resultado encontrado. A DBP pode ser definida por meio de características clínicas, radiológicas e histológicas próprias. Acomete, em geral, os recém-nascidos prematuros submetidos à oxigenoterapia e ventilação mecânica nos primeiros dias de vida, sendo um problema ainda prevalente no mundo todo ${ }^{30}$. Desta forma, uma revisão de literatura, publicada no ano de 2008, observou que as medidas de função respiratória das crianças com DBP revelaram-se menores do que o previsto e inferiores quando comparados aos grupos controles ${ }^{31}$. Além disso, um estudo Vom Hove et al. ${ }^{32}$ demonstrou que as crianças com DBP foram mais propensas a realizar o tratamento com medicação para controlar a asma em comparação aos sujeitos hígidos.

Por outro lado, menos de $1 / 3$ dos estudos incluídos nessa revisão demonstrou valores normais de espirometria em idade escolar no grupo de crianças nascidas prematuras e com baixo peso $6,17,25$. Tais autores atribuem esses resultados ao fato das crianças recuperarem ou minimizarem as sequelas pulmonares devido ao crescimento pulmonar e nutricional adequado ao longo da infância, reduzindo ou tornando menos evidentes as lesões pulmonares. Esses achados conflitantes quanto à preservação ou comprometimento da função pulmonar dessa população na idade escolar podem ser atribuídos às diferentes metodologias utilizadas, como distintos delineamentos, critérios de seleção e inclusão dos grupos avaliados, além da ausência da avaliação dos fatores sócio-demográficos e ambientais ${ }^{29}$. Por fim, os artigos incluídos também foram heterogêneos em relação aos critérios de inclusão para a idade gestacional e peso ao nascimento, tanto no grupo estudado como no controle.

O fato de não ter sido encontrado nenhum estudo nacional que objetivasse avaliar a influência da prematuridade/baixo peso ao nascimento sobre a função pulmonar na idade escolar, evidencia uma carência de informações sobre esses fatores a nível nacional. Além disso, deve-se estimular a identificação de subgrupos de risco para o desenvolvimento de uma pior função pulmonar no decorrer da vida através de avaliações longitudinais, visando minimizar o impacto do nascimento precoce.

Em conclusão, os achados da presente revisão de literatura demonstram que a maioria dos estudos incluídos sugere uma influência negativa da prematuridade e do baixo peso ao nascimento sobre a função pulmonar na idade escolar. Todavia, a interpretação destes resultados pode ter sido prejudicada devido às divergências quanto às metodologias e aos critérios utilizados pelos estudos incluídos.

\section{REFERÊNCIAS}

1. Santos D, Campos D, Gonçalves V, Mello B, Campos T, Gagliardo $\mathrm{H}$. Influence of the low birth weight on motor performance of term infants in the first semester of life. Braz J Phys Ther 2004;8(3):261-66.

2. da Silva ES, Nunes ML. The influence of gestational age and birth weight in the clinical assessment of the muscle tone of healthy term and preterm newborns. Arq Neuropsiquiatr 2005;63(4):956-62 http://dx.doi.org/10.1590/S0004-282X2005000600010

3. Coelli AP, Nascimento LR, Mill JG, Molina MeC. Preterm birth as a risk factor for high blood pressure in children: a systematic review. Cad Saude Publica 2011;27(2):207-18. 
4. Doyle LW, Group VICS. Evaluation of neonatal intensive care for extremely low birth weight infants in Victoria over two decades: I. Effectiveness. Pediatrics 2004;113(3Pt 1):505-9. http://dx.doi. org/10.1542/peds.113.3.505

5. Eber E, Zach MS. Long term sequelae of bronchopulmonary dysplasia (chronic lung disease of infancy). Thorax 2001;56(4): 317-23. http://dx.doi.org/10.1136/thorax.56.4.317

6. Kitchen WH, Olinsky A, Doyle LW, Ford GW, Murton LJ, Slonim L, Callanan C. Respiratory health and lung function in 8-yearold children of very low birth weight: a cohort study. Pediatrics 1992;89(6 Pt 2):1151-8.

7. Hoo AF, Dezateux C, Henschen M, Costeloe K, Stocks J. Development of airway function in infancy after preterm delivery. J Pediatr 2002;141(5):652-8. http://dx.doi.org/10.1067/mpd.2002.128114

8. Greenough A. Late respiratory outcomes after preterm birth. Early Hum Dev 2007;83(12):785-8. http://dx.doi.org/10.1016/j. earlhumdev.2007.09.006

9. Siltanen M, Savilahti E, Pohjavuori M, Kajosaari M. Respiratory symptoms and lung function in relation to atopy in children born preterm. Pediatr Pulmonol 2004;37(1):43-9. http://dx.doi. org/10.1002/ppul.10402

10. Rona RJ, Gulliford MC, Chinn S. Effects of prematurity and intrauterine growth on respiratory health and lung function in childhood. BMJ 1993;306(6881):817-20. http://dx.doi.org/10.1136/ bmj.306.6881.817

11. Kilbride HW, Gelatt MC, Sabath RJ. Pulmonary function and exercise capacity for ELBW survivors in preadolescence: effect of neonatal chronic lung disease. J Pediatr 2003;143(4):488-93. http://dx.doi. org/10.1067/S0022-3476(03)00413-X

12. Doyle LW, Chavasse R, Ford GW, Olinsky A, Davis NM, Callanan C. Changes in lung function between age 8 and 14 years in children with birth weight of less than 1,501 g. Pediatr Pulmonol 1999;27(3):185-90. http://dx.doi.org/10.1002/(SICI)10990496(199903)27:3<185::AID-PPUL6>3.0.CO;2-0

13. Maritz GS, Morley CJ, Harding R. Early developmental origins of impaired lung structure and function. Early Hum Dev 2005; 81(9):763-71. http://dx.doi.org/10.1016/j.earlhumdev.2005.07. 002

14. McLeod A, Ross P, Mitchell S, Tay D, Hunter L, Hall A, Paton J, Mutch L. Respiratory health in a total very low birthweight cohort and their classroom controls. Arch Dis Child 1996;74(3):188-94. http://dx.doi.org/10.1136/adc.74.3.188

15. Jobe $\mathrm{AH}$. An unknown: lung growth and development after very preterm birth. Am J Respir Crit Care Med 2002;166(12 Pt 1):1529-30. http://dx.doi.org/10.1164/rccm.2209012

16. Chan KN, Wong YC, Silverman M. Relationship between infant lung mechanics and childhood lung function in children of very low birthweight. Pediatr Pulmonol 1990;8(2):74-81. http://dx.doi. org/10.1002/ppul.1950080204

17. Lercher P, Schmitzberger R. Birth weight, education, environment, and lung function at school age: a community study in an alpine area. Eur Respir J 1997;10(11):2502-7. http://dx.doi.org/10.1183/0 9031936.97.10112502

18. Schraeder BD, Czajka C, Kalman DD, McGeady SJ. Respiratory health, lung function, and airway responsiveness in school-age survivors of very-low-birth-weight. Clin Pediatr (Phila) 1998;37(4):237-45. http://dx.doi.org/10.1177/000992289803700404

19. Wjst M, Popescu M, Trepka MJ, Heinrich J, Wichmann HE. Pulmonary function in children with initial low birth weight. Pediatr Allergy Immunol 1998;9(2):80-90. http://dx.doi. org/10.1111/j.1399-3038.1998.tb00308.x

20. Kennedy JD, Edward LJ, Bates DJ, Martin AJ, Dip SN, Haslam RR, McPhee AJ, Staugas RE, Baghurst P. Effects of birthweight and oxygen supplementation on lung function in late childhood in children of very low birth weight. Pediatr Pulmonol 2000;30(1):32-40. http://dx.doi.org/10.1002/1099-0496(200007)30:1<32::AIDPPUL6>3.0.CO;2-9

21. Korhonen P, Laitinen J, Hyödynmaa E, Tammela O. Respiratory outcome in school-aged, very-low-birth-weight children in the surfactant era. Acta Paediatr 2004;93(3):316-21. http://dx.doi. org/10.1111/j.1651-2227.2004.tb02954.x

22. Doyle LW, Group VICS. Respiratory function at age 8-9 years in extremely low birthweight/very preterm children born in Victoria in 1991-1992. Pediatr Pulmonol 2006;41(6):570-6. http://dx.doi. org/10.1002/ppul.20412

23. Palta M, Sadek M, Barnet JH, Evans M, Weinstein MR, McGuinness G, Peters ME, Gabbert D, Fryback D, Farrell P. Evaluation of criteria for chronic lung disease in surviving very low birth weight infants. Newborn Lung Project. J Pediatr 1998;132(1):57-63. http://dx.doi. org/10.1016/S0022-3476(98)70485-8

24. Smith LJ, van Asperen PP, McKay KO, Selvadurai H, Fitzgerald DA. Reduced exercise capacity in children born very preterm. Pediatrics 2008;122(2):e287-93. http://dx.doi.org/10.1542/peds.2007-3657

25. Qi-Qiang H, Tze-Wai W, Lin D, Zhuo-Qin J, Yang G, Guo-Zhen L, Ignatius YT. Birth weight and lung function in a cohort of Chinese school children. Pediatr Pulmonol 2009;44(7):662-8. http://dx.doi. org/10.1002/ppul.21035

26. Cazzato S, Ridolfi L, Bernardi F, Faldella G, Bertelli L. Lung function outcome at school age in very low birth weight children. Pediatr Pulmonol 2013;48(8):830-7. http://dx.doi.org/10.1002/ppul. 22676

27. Hacking DF, Gibson AM, Robertson C, Doyle LW, (VICS) VICSG. Respiratory function at age 8-9 after extremely low birthweight or preterm birth in Victoria in 1997. Pediatr Pulmonol 2013;48(5): 449-55. http://dx.doi.org/10.1002/ppul.22619

28. Elder DE, Hagan R, Evans SF, Benninger HR, French NP. Recurrent wheezing in very preterm infants. Arch Dis Child Fetal Neonatal Ed 1996;74(3):F165-71. http://dx.doi.org/10.1136/fn.74.3.F165

29. Friedrich $\mathrm{L}$, Corso $\mathrm{AL}$, Jones $\mathrm{MH}$. Pulmonary prognosis in preterm infants. J Pediatr 2005;81(1):78-88.

30. Monte L, Silva Filho L, Miyoshi M, Rozov T. Bronchopulmonary dysplasia. J Pediatr (Rio J) 2005;81(99-110).

31. Cabral LA, Velloso M. Impacto da displasia broncopulmonar na função pulmonar de crianças em idade escolar. Ciência em Mov 2008:88-100.

32. Vom Hove M, Prenzel F, Uhlig HH, Robel-Tillig E. Pulmonary outcome in former preterm, very low birth weight children with bronchopulmonary dysplasia: a case-control follow-up at school age. J Pediatr 2014;164(1):40-5.e4. http://dx.doi.org/10.1016/j. jpeds.2013.07.045 